\title{
Usabilidade e experiência do usuário de sistemas de informação: em busca de limites e relações
}

\author{
Usability and User experience of information systems: in search of boundaries and \\ relationships
}

\author{
Maria Aniolly Queiroz Maia \\ Doutoranda em Gestão e Organização do Conhecimento \\ Universidade Federal de Minas Gerais \\ aniollymaia@gmail.com
}

Ricardo Rodrigues Barbosa

Doutor em Administração de Empresas

Universidade Federal de Minas Gerais

rrbarb@gmail.com

Peter Williams

PhD em Estudos em Informação

University College London

peter.williams@ucl.ac.uk

Resumo

A usabilidade e a experiência do usuário de sistemas de informação são aspectos de grande relevância no processo de desenvolvimento de interfaces digitais. Cada um desses elementos contribui, objetiva e/ou subjetivamente, para a identificação de aspectos fundamentais para a criação de interfaces de sistemas de informação. Nessa perspectiva, o referido estudo tem o objetivo de apresentar reflexões acerca dos possíveis limites e relações entre esses dois elementos. Trata-se de uma pesquisa qualitativa, baseada em pesquisa bibliográfica sobre as temáticas usabilidade e experiência do usuário em bases de dados internacionais (Scopus e Web of Science), sem delimitação de área do conhecimento e recorte temporal, destacando, especialmente, os termos de forma relacional. A partir do referido estudo, foi possível evidenciar a carência de estudos capazes de unificar o significado desses conceitos e, consequentemente, promover o avanço e o aprofundamento de estudos dessas temáticas. Assim, o estudo evidenciou que a usabilidade e a experiência do usuário possuem numerosas características, o que salienta a necessidade de um entendimento mais aprofundado por parte dos pesquisadores interessados nas referidas temáticas, devido à ausência de consenso sobre essas questões.

\section{Palavras-chave}

Experiência do usuário. Interfaces digitais. Usabilidade.

\begin{abstract}
The usability and user experience of information systems are very important aspects in the development process of digital interfaces. Each of these elements contributes, objective and/or subjectively, for the identification of fundamental aspects for creating interfaces to information systems. In this perspective, the study aims to present reflections on the possible limits and relations between these two elements. It is a qualitative research, based in bibliographic research on the Usability and user experience theme in international databases (Scopus and Web of Science), without delimitation of area of knowledge and temporal, highlighting especially the terms of relational form. From this study, it was possible to highlight the lack of studies able to unify the meaning of these concepts and, consequently promoting the advancement and deepening of these thematic studies. Thus, the study showed that Usability and User experience have numerous features, which stresses the need for a deeper understanding on the part of researchers interested in these topics, due to the absence of consensus on these issues.
\end{abstract}

Keywords

User experience. Digital interfaces. Usability.

DOI: $10.28998 /$ cirev.2019v6n3c

Este artigo está licenciado sob uma Licença Creative Commons 4.0

Submetido em: $21 / 08 / 2019$

Aceito em: $11 / 11 / 2019$

Publicado em: 07/01/2020 


\section{INTRODUÇÃO}

Com o crescente volume de conteúdos disponíveis na rede mundial de computadores, emerge uma preocupação com as formas de organização, acesso e uso da informação. Dessa forma, observa-se que o uso da informação disponível na Internet relaciona-se com a satisfação e experiência dos usuários, bem como a eficiência e eficácia das interfaces dos sistemas de informação. Dessa forma, a usabilidade e a experiência do usuário, destacam-se como aspectos fundamentais para o atendimento às necessidades dos usuários quanto à melhoria das interfaces digitais.

Neste contexto, acredita-se ser relevante analisar as diversas definições de usabilidade e experiência do usuário adotadas nas mais diversas áreas do conhecimento, preocupadas com as diversas formas de garantia de acesso, recuperação e uso da informação, por acreditar que assim é possível colher elementos capazes de contribuir para o desenvolvimento de interfaces cada vez melhores e, principalmente, voltadas às reais necessidades dos seus usuários.

Levando em conta os elementos acima apontados, o presente estudo objetiva apresentar reflexões acerca dos limites e relações entre essas duas temáticas, na medida em que podem ser considerados termos divergentes e/ou complementares. Nessa perspectiva, o estudo é de natureza qualitativa e se baseou em um levantamento bibliográfico, em fontes de informação internacionais, notadamente a Scopus e Web of Science, disponível no Portal de Periódicos da Coordenação de Aperfeiçoamento de Pessoal de Nível Superior (CAPES), sem delimitação de área do conhecimento e recorte temporal.

Assim, o artigo em foco explora características da produção científica acerca dos temas: usabilidade e experiência do usuário, de forma isolada e relacional, com vistas a buscar os limites e relações entre essas temáticas, e assim identificar possíveis avanços para as áreas do conhecimento preocupadas com as necessidades dos usuários diante do cenário das interfaces dos sistemas de informação digitais, que crescem a cada dia, com vistas a facilitar as atividades desenvolvidas pelos membros sociais e organizações.

\section{USABILIDADE}

A palavra "usabilidade", na sua forma mais fundamental, é simplesmente a forma substantiva do adjetivo "utilizável", e se refere a uma propriedade ou característica de um artefato tecnológico; ou seja, em que medida um sistema é utilizável. (BEST; SMYTH, 2011).

Em se tratando da perspectiva da usabilidade como propriedade ou característica de um sistema ser ou não utilizável, destaca-se que o termo tem suas raízes na Ciência Cognitiva, tendo sido usado inicialmente na década de 1980 nas áreas de Psicologia e Ergonomia, quando houve um aumento considerável na quantidade de pesquisadores preocupados com o desenvolvimento de sistemas fáceis de usar. Na ocasião, user friendly era o termo utilizado em referência aos produtos e/ou sistemas com facilidade de uso. Ao passar dos anos, mas ainda na referida década, este termo foi alterado para a palavra usability, sendo Jakob Nielsen um dos principais divulgadores desse novo termo. (SANTA ROSA; PEREIRA JUNIOR; LAMEIRA, 2016).

Shackel (2009, p. 340, tradução nossa, grifo do autor) acrescenta que:

A definição de usabilidade provavelmente foi tentada pela primeira vez por Miller (1971) em termos de medidas para "facilidade de uso", e estas foram desenvolvidas por Bennett (1979) para descrever a usabilidade. O conceito de usabilidade foi 
discutido em primeiro lugar e uma definição formal detalhada [...] foi tentado por Shackel (1981), e Bennett (1984) que modificou e desenvolveu a definição.

Conforme Galitz (2002), Bennett foi o primeiro a usar o termo usabilidade para descrever a eficácia do desempenho humano no uso de um sistema de informação. Já nos anos seguintes, uma definição mais formal foi proposta por Shackel e modificado por Bennett. No ano de 1991, Shackel definiu usabilidade como "[...] a capacidade de ser usada por humanos de forma fácil e eficaz." (GALITZ. 2002, p. 55).

Inúmeras são as definições do termo usabilidade, umas relacionadas às características ergonômicas de um determinado produto; outras orientadas ao usuário, identificando a relevância da minimização de esforço mental por parte desse, e ainda, a atitude desse indivíduo diante do uso de um produto; outras abordagens, com enfoque no desempenho e interação do usuário frente a um produto e/ou interface; e também, definições relacionadas ao contexto de uso. (DIAS, 2006).

Embora a usabilidade tenha um papel cada vez mais importante na Interação Humano Computador $(\mathrm{HCl})$, não existe uma definição universalmente aceita. De fato, os pesquisadores têm tentado, individualmente, capturar e definir a essência desse conceito, mas sem alcançar consenso. (CHEN; GERMAIN; RORISSA, 2011). A esse respeito, Pack (2003, tradução nossa) conclui que "[...] o termo tem sido usado tantas vezes em tantos contextos que se corre o risco de perder seu significado preciso.".

Em um contexto de diversas definições de usabilidade, há também iniciativas institucionais no sentido de dirimir ambiguidades a esse respeito. De acordo com a ISO/IEC 9126 (1991, p. 3, tradução nossa), usabilidade se refere a "[...] um conjunto de atributos de software que evidenciam o esforço necessário para seu uso e para a avaliação individual de tal uso por determinado conjunto de usuários.". Nesse caso, sua abordagem está relacionada ao produto e ao usuário. Ainda no ano de 1991, foi criada a Usability Professionals Association (UPA), que no ano de 2012 passou a ser denominada de User Experience Professionals Association (UXPA). Trata-se de uma associação de profissionais da experiência do usuário que oferece suporte a pessoas que pesquisam, projetam e avaliam a experiência do usuário de produtos e serviços, assim como é uma organização escolhida por profissionais de usabilidade em todo o mundo. (USABILITY PROFESSIONALS ASSOCIATION, 2002).

Já a NBR 9241 - 11 destaca que a "usabilidade é a medida na qual um produto pode ser usado por usuários específicos para alcançar objetivos específicos com eficácia, eficiência e satisfação em um contexto específico de uso" (ASSOCIAÇÃO BRASILEIRA DE NORMAS TÉCNICAS, 2002, p. 3).

A usabilidade também pode ser considerada como a qualidade de uso de um produto e/ou sistema a partir das necessidades de um determinado usuário. Esta característica depende de tarefas específicas que os usuários realizam com o sistema e de fatores relacionados ao ambiente físico, tais como: iluminação, ruídos, interrupções de tarefas e disposição de equipamentos. Ou seja, qualquer alteração em um aspecto relevante do contexto de uso pode comprometer a usabilidade de determinado sistema. (DIAS, 2006).

Apesar da dificuldade no que diz respeito à personalização do conceito de usabilidade, sabe-se que esta característica traz inúmeros potenciais benefícios no contexto do desenvolvimento de interfaces. Dessa forma, Bevan (1998) aponta os seguintes benefícios, como sendo: 
- maior eficiência: um sistema que incorpora um bom design ergonômico e adaptado à maneira preferida de trabalhar, permitirá que o usuário opere de maneira eficaz e eficiente;

- melhor produtividade: uma boa interface para um produto bem projetado permitirá ao usuário concentrar-se na tarefa e não na ferramenta que, se concebida de forma inadequada, pode estender ao invés de reduzir o tempo de realização de uma tarefa, assim como afetar diretamente outros aspectos como desempenho e qualidade;

- redução de erros: uma proporção significativa do chamado "erro humano" pode ser atribuída a um produto com uma interface projetada para uma funcionalidade não condizente com as necessidades do usuário. Ao se evitar inconsistências, ambiguidades ou outras falhas de design de interface, é possível minimizar os erros do usuário;

- redução de treinamentos: uma interface de usuário e diálogos mal projetados podem representar barreiras ao desenvolvimento de um sistema tecnicamente sólido. Um sistema bem projetado com foco no usuário final pode reforçar o aprendizado, reduzindo o tempo e o esforço de treinamento;

- melhor aceitação: usuários preferem usar e é mais provável que confiem em um sistema bem projetado com acesso à funcionalidades que tornem a informação fácil de encontrar, assimilar e usar.

Além dos benefícios já mencionados, pode-se dizer que a usabilidade é um atributo que traz contribuições para a qualidade de um produto, principalmente, no que se refere ao aspecto relacionado à minimização do tempo do usuário. "Tempo é algo precioso a todo ser humano. Em geral, as pessoas tornam-se insatisfeitas, frustradas e até mesmo enraivecidas quando não conseguem executar atividades de maneira fácil e rápida." (SILVA FILHO, 2010, p. 13).

De acordo com Nielsen (1993), a usabilidade de um sistema tem múltiplas qualidades, sendo estas: fácil de aprender, eficiente de usar, fácil de lembrar, apresenta baixa taxa de erro com zero erro catastrófico e satisfação do usuário. Adicionalmente, Nielsen (1993) apresentou dez heurísticas de métodos de inspeção de usabilidade, por considerá-las essenciais para o desenvolvimento de sistemas interativos. Nesse caso, os usuários exercem papel fundamental frente aos sistemas de informação, podendo personalizar a interface, dar feedback e adaptar o sistema conforme suas necessidades. Assim, a participação dos usuários pode contribuir para o desenvolvimento e manutenção dos sistemas de informação oferecidos à sociedade.

As heurísticas de Nielsen (1993) contemplam os seguintes aspectos:

- visibilidade de status do sistema: destaca a necessidade do usuário ser informado pelo sistema sobre o que está acontecendo, em tempo razoável;

- relacionamento entre a interface do sistema e o mundo real: aborda a necessidade de o modelo lógico do sistema ser compatível com o modelo lógico do usuário;

- liberdade e controle do usuário: indica que o sistema deve tornar disponíveis funções que possibilitem desfazer ou refazer uma determinada ação, assim como, retornar ao caminho anterior caso necessário; 
- consistência e padronização: dizem respeito à necessidade de o sistema ser consistente quanto à utilização de sua simbologia e à sua plataforma de hardware e software;

- prevenção de erros: indica que o design do sistema deve ser projetado para informar o usuário que alguma ação o induzirá ao erro, podendo esse ser efetuado por engano (pretensão de realização de uma ação efetuando outra) ou deslize (compreensão de alguma informação equivocada ou entendida de outra forma);

- reconhecimento ao invés de lembrança: destaca que, para o bom funcionamento de um sistema, as instruções devem estar visíveis no contexto em que o usuário se encontra;

- flexibilidade e eficiência de uso: indica que o sistema deve prever o nível de proficiência do usuário em relação ao próprio sistema;

- estética e design minimalista: trata que os diálogos do sistema devem conter somente informações relevantes ao seu funcionamento;

- ajuda aos usuários: no reconhecimento, diagnóstico e correção de erros apontam que as mensagens devem ser expressas em linguagem clara e indicar possíveis soluções; e

- ajuda e documentação: infere que a informação desejada deve ser facilmente encontrada, preferencialmente contextualizada e não muito extensa.

Para Dumas e Redish (1999) usabilidade significa que os usuários de um produto devem concluir, de forma rápida e de maneira fácil, suas tarefas. Sua definição se apoia em quatro pontos:

1. Usabilidade significa foco no usuário - para se desenvolver um produto usável, é necessário conhecer, entender e trabalhar com pessoas que representam usuários atuais ou potenciais do produto;

2. Pessoas que utilizam o produto para serem produtivos - pessoas consideram um produto fácil de aprender e usar com relação ao tempo que levam para fazer o que elas querem; o número de passos que elas vão atravessar; e o sucesso na antecipação de qual ação adotar;

3. Usuários são pessoas ocupadas tentando concluir tarefas - pessoas correlacionam usabilidade com produtividade;

4. Usuários decidem quando um produto é fácil de usar - usuários, não os designers e nem desenvolvedores, determinam quando um produto é fácil de utilizar.

Embora os sistemas de informação tenham passado por importantes transformações, e ainda, tenha ocorrido uma mudança nas atitudes dos usuários, persiste a necessidade de um contínuo aprimoramento conceitual do termo usabilidade, de modo a contemplar o contexto dos dispositivos móveis, sendo estes, recursos cada vez mais utilizados diariamente pelas pessoas.

A necessidade de tal evolução pode ser justificada a partir da identificação da evolução dos sistemas de informação, conforme alguns marcos importantes, tais como: o surgimento dos primeiros sistemas de informação computadorizados, que originalmente eram usados apenas por desenvolvedores; o desenvolvimento de sistemas de computadores pessoais (Apple, IBM - International Business Machines Corporation, entre outros), onde foi possível uma maior participação dos usuários; posteriormente, houve a criação de interfaces 
gráficas mais amigáveis, culminando o desenvolvimento de sistemas móveis e na participação mais ativa dos usuários.

Assim, percebe-se a necessidade de melhorias não apenas no que se refere ao conceito usabilidade, mas também nas formas de avaliação desse aspecto tão relevante para as organizações e sociedade em geral. Nesse sentido, sendo a interface um componente essencial de um sistema utilizável, Dee e Allen (2006) consideram que o aspecto cognitivo do processamento da informação por parte do usuário também deve ser considerado ao se desenvolver uma interface de dispositivos móveis ou desktops. Segundo esses autores, focar apenas na interface é uma visão muito restrita da usabilidade, uma vez que a coleta de informações em um ambiente digital é um processo dinâmico de interação humana com sistemas de informação.

Desta forma, percebe-se que o processo de avaliação de usabilidade de interface vai além da análise de interação entre o usuário e a máquina; ela envolve uma série de aspectos relacionados à cognição humana que influencia consideravelmente na avaliação de usabilidade.

\section{EXPERIÊNCIA DO USUÁRIO (USER EXPERIENCE)}

De acordo com Alben (1996), a experiência do usuário com um sistema de informação diz respeito a todos os aspectos de como as pessoas usam um determinado produto, tais como: a maneira como eles sentem em suas mãos, quão bem eles entendem como funciona, como eles se sentem enquanto estão usando, se o produto serve bem aos seus propósitos e como se encaixa em todo o seu contexto de uso.

Na década de 1990, Donald Norman, que nesta época atuava como Vice-Diretor da empresa Apple, foi um dos primeiros autores a mencionar o termo "Experiência do usuário" para descrever todos os aspectos de uma experiência da pessoa com um sistema. (NORMAN; MILLER; HENDERSON, 1995). Norman introduziu o referido termo por acreditar que a "usabilidade" fosse estreita demais para representar uma visão holística de interações humano-computador.

Para a ISO 9241-210 (2010) experiência do usuário está relacionada às percepções e respostas das pessoas que resultam do uso ou da expectativa de uso de um produto, sistema ou serviço. A referida experiência engloba aspectos relacionados a emoção, crença, preferência, percepção, resposta física e psicológica, comportamento e ações realizadas antes, durante e após o uso de um determinado artefato.

Conforme Vasconcelos (2007, p. 25), "A experiência do usuário influi na qualidade e na satisfação que alguém tem em interagir com algum produto, serviço ou sistema. Muitas vezes o sucesso de um produto é definido pela qualidade da experiência antes, durante e depois de seu uso.".

A propósito das contribuições da experiência do usuário nos estudos de interação entre os indivíduos e a tecnologia, Cybis, Betiol e Faust (2010, p. 365) discorrem:

[...] a definição de experiência do usuário surge na área de interação homemtecnologia com o objetivo de proporcionar uma visão mais abrangente das relações entre as propriedades funcionais, estéticas e de interação do produto, assim como a maneira pela qual os indivíduos respondem a elas quanto aos aspectos, físico, cognitivo e emocional. 
Essa experiência pode ser positiva ou negativa. Isso dependerá da percepção do usuário, e ainda, do sentimento do sujeito para com o produto e/ou interface no processo de interação. A experiência do usuário engloba diversos aspectos apresentados como facetas por Morville (2004), conforme descrição que segue:

- útil (useful): desenvolver produtos e sistemas úteis, devendo ser aplicadas soluções com aprimoramento contínuo;

- utilizável (usable): oferecer facilidade de utilização continua; a usabilidade é necessária, mas não suficiente para contemplar as necessidades do usuário;

- desejável (desirable): utilizar elementos de design emocional, como, imagem, marca que dão poder e valor de identidade;

- encontrável (findable): oferecer navegação e objetos localizáveis permitindo ao usuário encontrar o que precisa;

- acessível (acessible): atender a usuários com ou sem deficiência;

- credível (credible): conquistar a confiança do usuário com relação ao ambiente e ao conteúdo apresentado;

- valioso (valuable): oferecer valor aos patrocinadores, de modo a atender à satisfação do usuário; valorar a missão para organizações sem fins lucrativos.

As facetas acima, se atendidas de forma eficiente e eficaz às necessidades do usuário, garantem uma boa experiência aos indivíduos. Se, ao contrário, algumas dessas facetas não atenderem às suas necessidades, a experiência com o uso do sistema fica prejudicada.

Acredita-se ser relevante destacar que para a promoção da acessibilidade, o sistema de informação deve ser percebível (estrutura, consistência, conteúdo alternativo), interativo (navegação, feedback, controle e segurança, independência de dispositivo), navegável (navegação, busca, controle e segurança) e compreensível (estrutura, navegação, consistência). (W3C, 2002).

O conceito de experiência do usuário é recente e com uma maior amplitude quando relacionada à usabilidade. A criação do referido termo pautou-se na justificativa de que a usabilidade está fortemente ligada aos fatores maioritariamente instrumentais, sendo estes relacionados à eficiência da tarefa e/ou trabalho. Nesse sentido, a experiência do usuário surge como uma perspectiva mais abrangente no que tange à qualidade de uma interface e/ou produto. Esse termo concentra-se na interação entre pessoas e produtos, e todos os tipos de experiências que resultam disso, como é o caso dos aspectos físicos, emocionais, cognitivos e estéticos. (HAAKSMA; JONG; KARREMAN, 2018).

Assim como a usabilidade, o conceito de experiência do usuário engloba diversas críticas. Sendo a imprecisão, a principal delas. De acordo com Haaksma, Jong e Karreman, (2018, p. 119, tradução nossa) "[...] devido à sua amplitude, maleabilidade e complexidade do panorama da pesquisa, é difícil alcançar uma definição universal de UX.". De fato, trata-se de um termo com múltiplos significados, sem que haja uma definição consensual nem teoria coerente. (HASSENZAHL; TRACTINSKY, 2006).

De acordo com Law et al. (2009), a definição de experiência do usuário é um desafio para os pesquisadores. Para eles, algumas das razões para essa dificuldade se refere, em primeiro lugar, ao fato de experiência do usuário está associada a uma ampla gama de conceitos difusos e dinâmicos, incluindo conceitos emocionais, variáveis afetivas, experienciais, hedônicas e estéticas. Nesse sentido, a inclusão e exclusão de determinadas variáveis parecem arbitrárias, dependendo do autor, finalidade e interesse. Em segundo lugar, a unidade 
de análise para experiência do usuário é muito maleável, variando entre um único aspecto de uma interação individual por parte de um usuário final até a totalidade da natureza das interações de vários usuários finais com empresas que oferecem diversos serviços. Em terceiro lugar, o panorama da pesquisa sobre experiência do usuário é fragmentado e complicado por diversos modelos teóricos com diferentes focos como hedônica, entre outros.

\section{USABILIDADE E USER EXPERIENCE: EM BUSCA DE LIMITES E RELAÇÕES}

Com o intuito de atender ao objetivo de oferecer contribuições conceituais e promover algumas reflexões acerca dos possíveis limites e relações entre as temáticas usabilidade e experiência do usuário, foi realizado em janeiro de 2019, um levantamento da produção científica a esse respeito, a partir de buscas nas bases de dados Scopus e Web of Science. Como estratégia de busca, foram utilizados os termos "usability" e "user experience" de forma isolada, assim como a expressão "usability and user experience", em que o termo "and" não foi disposto como um operador booleano, mas uma palavra que também deveria ser pesquisada.

Em se tratando da busca à base Web of Science, a pesquisa compreendeu a busca por "título". Já no caso da Scopus, deu-se a partir do "título do artigo" (article title), pelo fato de não haver um campo de busca nomeado "título" na referida base. No que se refere à delimitação a partir do "título", esta foi utilizada pelo fato de acreditar que esse campo recuperaria de forma eficiente os documentos relacionados às temáticas apresentadas. Vale ressaltar que nas duas bases foi delimitada a tipologia documental referente a "artigos", sem restrição de tempo e área do conhecimento.

A estratégia de busca, a partir da utilização dos termos em separado e de forma conjunta (a partir de uma expressão), foi realizada com a intenção de visualizar o quantitativo de produções, notadamente a tipologia de artigos, existentes nas bases pesquisadas. A escoIha da expressão "usability and user experience" sem utilizar o "AND" como um operador booleano deu-se em razão de considerar que essa estratégia seria mais relevante para um refinamento dos artigos que apresentassem os dois termos no título.

Nessa direção, a Tabela 1 apresenta os resultados da pesquisa realizada a partir da estratégia de busca utilizada:

Tabela 1 - Resultado de busca em bases de dados

\begin{tabular}{lccc}
\hline \multicolumn{1}{c}{$\begin{array}{c}\text { Base de dados/ Tipo de } \\
\text { busca }\end{array}$} & $\begin{array}{c}\text { Termo 1 } \\
\text { "Usability" }\end{array}$ & $\begin{array}{c}\text { Termo 2 } \\
\text { "User experience" }\end{array}$ & $\begin{array}{c}\text { Expressão } \\
\text { "usability and user experience" }\end{array}$ \\
\cline { 2 - 4 } Scopus/ Título do artigo & 4.247 & 1.167 & 17 \\
Web of Science/ Título & 3.336 & 528 & 09 \\
\hline
\end{tabular}

Fonte: Elaborado pelos autores (2019).

Ao realizar a pesquisa na base de dados Scopus, foi possível visualizar que o termo usability possui registro a partir do ano de 1945, com o artigo de autoria de Dyer, H. S., intitulado "The usability of the concept of prejudice", publicado no periódico Psychometrika. No entanto, o referido estudo congrega a perspectiva conceitual da palavra preconceito, não havendo relação com a perspectiva da usabilidade de sistemas de informação em si. Identificou-se, ainda, que as produções relacionadas ao conceito de usabilidade foram impulsionadas no início da década de 1970, tendo um maior destaque no final dessa década. Já o termo user experience, teve um registro no ano de 1970 no periódico Journal of Chemical 
Documentation, cujo título é User experiences with primary journals on $16-\mathrm{Mm}$ Microfilm, e autoria de Starker, L. N.

O primeiro registro de artigo publicado no qual os termos usability and user experience apresentam-se como expressão ocorreu em 2006, no periódico Interactions, cujo título é Funcionality, usability, and user experience: three áreas of concern, autoria de McNamara, $\mathrm{N}$. e Kirakowski, J.

Conforme a base Scopus, há uma variedade de áreas do conhecimento que tratam a perspectiva da usabilidade relacionada à experiência do usuário, sendo que a Ciência da Computação se destaca com um total de $28,1 \%$ da produção científica publicada, conforme Tabela 2.

Tabela 2 - Área do conhecimento que trata a perspectiva da usabilidade aliada à experiência do usuário conforme publicações da Scopus

\begin{tabular}{lc}
\hline \multicolumn{1}{c}{ Área do conhecimento } & Porcentagem \\
\hline Ciência da Computação & $28,1 \%$ \\
Engenharias & $15,6 \%$ \\
Ciências Sociais & $15,6 \%$ \\
Negócios, Gestão e Contabilidade & $12,5 \%$ \\
Bioquímica, Genética e Biologia Molecular & $6,3 \%$ \\
Economia, Econometria e Finanças & $6,3 \%$ \\
Psicologia & $6,3 \%$ \\
Química & $3.1 \%$ \\
Medicina & $3,1 \%$ \\
Física & $3,1 \%$ \\
\hline
\end{tabular}

Fonte: Scopus (2019).

Na Tabela 2, destaca-se também a publicação de artigos classificados nas áreas de Ciências Sociais $(15,6 \%)$ e Engenharias (15,6\%), que, combinadas, têm se apropriado de maneira expressiva desses conceitos.

$\mathrm{Na}$ base de dados Web of Science, o primeiro registro de publicações relacionadas ao termo "usability" assim como o registro da Scopus, também foi realizado no ano de 1945 e com referência ao mesmo documento já mencionado, adicionado a publicação de título: The effect of imperfections on the usability of quartz for oscillator - plates, autoria de Lukesh, J. S., publicada no periódico American Mineralogist.

Ainda na Web of Science, foi realizada a consulta às produções de artigos na temática "user experience". Os primeiros registros, do ano 1966, são:

- DELAPLAI, I. J. Collaction and feed-back of user experience. Journal of the Royal Aeronautical Society, v. 70, n. 663. p. 415, 1966; e

- LOCKWOOD, B. Acting upon user experience - civil and military systems compared. Journal of the Royal Aeronautical Society, v. 70, n. 663, p. 418, 1966.

O primeiro artigo, no qual a expressão "usability and user experience" é apresentada, foi registrado na base Web of Science em 2008. A referência de tal documento é:

- YOON, SO-YEON; LAFFEY, JAMES; OH, HYUNJOO. Understanding usability and user experience of web-based 3D graphics technology. International Journal of human-computer interaction, v. 24, n. 3, p. 288-306, 2008. 
A identificação dos primeiros registros acerca das temáticas "usability and user experience" possibilitou o entendimento histórico dos referidos termos, notadamente, pelo fato de serem termos considerados adicionais, que surgem como resultado da evolução das necessidades dos usuários.

Após identificação dos registros históricos, foi realizada a leitura dos artigos (total de 26) que contemplam a expressão "usability and user experience" em seus títulos. Assim, a partir das produções analisadas, foi possível identificar que a usabilidade e a experiência do usuário são termos bastante relevantes para a maioria das áreas do conhecimento, e que mundialmente existem diversas pesquisas com enfoque nos referidos termos. No entanto, alguns pesquisadores procuram realizar a distinção, caracterização e identificação dos termos, de modo a avançar nas pesquisas e, consequentemente, trazer contribuições para o desenvolvimento de interfaces cada vez melhores sob a perspectiva das necessidades dos usuários.

Nessa perspectiva, percebeu-se que são múltiplas as interpretações referentes à relação da usabilidade com a experiência do usuário. Alguns estudos consideram a usabilidade uma parte da experiência do usuário, enquanto outras pesquisas tratam a usabilidade como condição para o sucesso da experiência do usuário. A esse respeito, destacam-se no Quadro 1 algumas das relações entre usabilidade e experiência do usuário, mencionadas e/ou descritas nos textos analisados.

Quadro 1 - Estudos que abordam relações da Usabilidade com a Experiência do usuário

\begin{tabular}{|c|c|}
\hline Autoria & Ideia central \\
\hline $\begin{array}{c}\text { Nielsen } \\
(1993,2008)\end{array}$ & $\begin{array}{l}\text { A usabilidade permite compreender se o sistema é bom o suficiente para satisfazer as } \\
\text { necessidades e exigências dos usuários e outras partes interessadas. E a experiência do } \\
\text { usuário, não só inclui a usabilidade, mas também aspectos cognitivos, socioculturais e } \\
\text { afetivos, considerados elementos positivos da experiência dos usuários em sua intera- } \\
\text { ção com os produtos, como a experiência estética ou desejo de reutilizar o produto. }\end{array}$ \\
\hline $\begin{array}{l}\text { McNamara e } \\
\text { Kirakowski } \\
(2006)\end{array}$ & $\begin{array}{l}\text { Usabilidade está relacionada à interação entre o usuário e o produto. Já a experiência } \\
\text { do usuário considera a relação mais ampla entre o produto e o usuário para investigar } \\
\text { a experiência pessoal do indivíduo em usá-lo. }\end{array}$ \\
\hline $\begin{array}{c}\text { Tullis e Albert } \\
(2008)\end{array}$ & $\begin{array}{l}\text { A usabilidade é considerada como a habilidade do usuário em realizar a tarefa com } \\
\text { sucesso. Já a experiência do usuário congrega uma visão mais ampla, que complemen- } \\
\text { ta a usabilidade, auxilia esta relação. A experiência do usuário foca na interação indivi- } \\
\text { dual como sentimentos, percepções e intenções resultantes desta interação. }\end{array}$ \\
\hline Bev & $\begin{array}{l}\text { Descreveu três visões do relacionamento entre experiência do usuário e usabilidade, } \\
\text { sendo estas: experiência do usuário como a elaboração do aspecto satisfação de usabi- } \\
\text { lidade; experiência do usuário como algo distinto de usabilidade; e experiência do } \\
\text { usuário como um termo genérico para todas as percepções e respostas dos usuários } \\
\text { (tanto subjetivas quanto objetivas). }\end{array}$ \\
\hline $\begin{array}{l}\text { Gegner, } \\
\text { Runonen e } \\
\text { Keinonen } \\
\text { (2011) }\end{array}$ & $\begin{array}{l}\text { Experiência do usuário não é apenas um termo muito divulgado, mas também um } \\
\text { contramovimento para o pensamento da usabilidade. Considerando que o significado } \\
\text { central da usabilidade lida, principalmente, com a interação humano-computador, } \\
\text { mais eficiente a experiência do usuário destaca os aspectos hedônicos de informática. }\end{array}$ \\
\hline $\begin{array}{l}\text { Kurosu } \\
(2015)\end{array}$ & $\begin{array}{l}\text { Experiência do usuário é atualmente a palavra de ordem, no entanto, o conceito de } \\
\text { usabilidade ainda é muito importante, mesmo que a conotação de experiência do usu- } \\
\text { ário seja muito mais ampla que a usabilidade. Para tanto, o autor caracteriza a relação } \\
\text { entre usabilidade e experiência do usuário, situando esta na qualidade de um artefato } \\
\text { em uso, e a usabilidade como um dos muitos aspectos da qualidade objetiva do artefa- } \\
\text { to. }\end{array}$ \\
\hline
\end{tabular}

Fonte: Elaborado pelos autores (2019). 
A partir das explanações acima, pode-se dizer que os aspectos objetivos estão especialmente associados com a usabilidade; no entanto, fatores subjetivos (como emoções e percepções) estão relacionados com a experiência do usuário. Assim, percebe-se que alguns aspectos da experiência do usuário estão além do controle dos projetistas de sistemas, uma vez que são elementos bastante subjetivos a serem identificados, na maioria das vezes, em ambientes controlados (salas, laboratórios, escritórios, dentre outros).

Usabilidade e experiência do usuário são consideradas parte do Design Centrado no Usuário, definido como "uma abordagem ao desenvolvimento de sistemas interativos cujo objetivo é tornar os sistemas utilizáveis e úteis" (ISO 9241-210, 2010, p. 6). Vale ressaltar que, antes da criação do padrão ISO 9241-210 (2010), utilizava-se o Padrão ISO 13407 (1999), que normatizava aspectos relacionados à usabilidade. No entanto, no ano de 2010 o referido documento (ISO 9241-210, 2010) foi atualizado para considerar o contexto da experiência do usuário.

A definição ISO 9241-210, referente à experiência do usuário, destaca que alguns critérios de avaliação de usabilidade podem ser usados para avaliar aspectos da experiência do usuário, ao destacar que a usabilidade pode incluir aspectos perceptivos e emocionais que são tipicamente associados à experiência do usuário. Essa abordagem corrobora alguns questionamentos referentes aos limites e relacionamentos entre os referidos conceitos. (HAAKSMA; JONG; KARREMAN, 2018).

Em seu trabalho sobre a demarcação conceitual da experiência do usuário, Law e Schaik (2010) enfatizam que os métodos, as técnicas e as ferramentas de avaliação e medição da experiência do usuário, em sua maioria, são extraídos dos aspectos utilizados no processo de avaliação de usabilidade. E que as ferramentas (métodos, métricas e fatores), utilizadas no processo de avaliação da experiência do usuário, têm como objetivo verificar a interação do usuário com o produto durante a realização de tarefas, e determinar, de forma objetiva, mas principalmente subjetiva, o grau da experiência dos usuários ao utilizar o produto. (ARAÚJO, 2014).

A esse respeito, Araújo (2014) acrescenta que os métodos, tanto de avaliação de usabilidade quanto da experiência do usuário, podem ser considerados complementares, considerando que a usabilidade está inclusa na experiência do usuário. De fato, dependendo dos fatores e métricas selecionadas, os métodos de avaliação da usabilidade podem ainda ser utilizados para avaliar os fatores hedônicos da experiência do usuário. Para tanto, faz-se necessário a identificação da fase do processo de desenvolvimento do sistema e escolha do melhor método a ser aplicado, a fim de garantir uma experiência de uso efetiva.

\section{CONSIDERAÇÕES FINAIS}

A usabilidade e a experiência do usuário contribuem sobremaneira para a melhoria das interfaces adotadas nos mais diversos equipamentos que têm como função organizar, recuperar e utilizar a informação, bem como possibilitar a comunicação das pessoas com tais equipamentos. Percebe-se, desta forma, que o entendimento de cada um dos termos se torna essencial para a identidade e o avanço das diversas áreas do conhecimento que abordam tais questões. $O$ desenvolvimento nessas áreas representa desafios para os pesquisadores, no sentido de contribuir para um maior entendimento dos diversos aspectos relacionados com a experiência dos usuários de sistemas e produtos de informação.

Inúmeras pesquisas apontam a usabilidade como um aspecto relevante para a experiência do usuário, e que este termo foi desenvolvido a partir da necessidade de identifica- 
ção de aspectos relevantes do processo de desenvolvimento de interfaces, que não poderiam ser contemplados exclusivamente com base nos atributos da usabilidade.

Nessa perspectiva, a partir de estudos iniciais realizados neste artigo, foi possível identificar que os dois termos (usabilidade e experiência do usuário) ainda passam por um processo de conceituação. E que ainda não existem estudos que destaquem todos os limites e relações entre os referidos termos.

É pertinente destacar a existência de numerosas técnicas e métodos de avaliação de usabilidade, alguns dos quais também são usados para avaliar a experiência do usuário. Acredita-se que esta situação pode dificultar o processo de entendimento dos limites e relações entre os termos em destaque. No entanto, corrobora a linha de entendimento referente à usabilidade ser um subconjunto da experiência do usuário.

A criação de novos padrões e mudanças de nomenclatura na associação entre usabilidade e experiência do usuário, que inicialmente priorizavam o contexto de usabilidade, suscitam questionamentos relacionados às relações e limitações de cada conceito. Mesmo quando se identificam diversas distinções entre os termos, ainda persistem problemas conceituais e metodológicos para os pesquisadores das mais diversas áreas do conhecimento que fazem uso de tais termos.

Enfatiza-se que não foi intenção deste estudo identificar a produção científica internacional, no que se refere ao relacionamento do conceito de usabilidade com a experiência do usuário. No entanto, com base na estratégia de busca utilizada, especialmente o uso da expressão "usability and user experience", foi possível evidenciar a necessidade de mais estudos que explorem tais relacionamentos.

A esse respeito, destaca-se que, ao utilizar em um dos critérios de busca o termo "AND" como uma palavra integrante da expressão "usability and user experience" delimitouse ainda mais a pesquisa realizada. Se tivesse sido realizada uma busca com a utilização dos dois termos (usability e user experience) mediados pelo operador booleano (AND) os resultados seriam diferentes, possivelmente mais abrangentes, no entanto, menos objetivos.

Por fim, reforça-se a necessidade de mais pesquisas que abordem os limites e relações entre usabilidade e experiência do usuário, em complementação aos numerosos estudos que evidenciam a experiência do usuário como um termo "abrangente", relacionado a aspectos subjetivos como sentimentos e percepções dos usuários ao usarem uma determinada interface, e as produções que enfatizam que a usabilidade se refere a um termo "limitado" ao processo de identificação da facilidade de uso no que diz respeito à eficiência, eficácia e satisfação.

\section{REFERÊNCIAS}

ALBEN, L. Quality of experience: defining the criteria for effective interaction design. Interactions, v. 3, n. 3, p. 11-15, 1996.

ARAÚJO, F. S. Avaliação da experiência do usuário: uma proposta de sistematização para o processo de desenvolvimento de produtos. 2014. 188 f. Tese (Doutorado em Engenharia de Produção) - Programa de Pós-graduação em Engenharia de Produção, Universidade Federal de Santa Catarina, Florianópolis, 2014. 
ASSOCIAÇÃO BRASILEIRA DE NORMAS TÉCNICAS. NBR: 9241-11: Requisitos Ergonômicos para Trabalho de Escritórios com Computadores: parte 11 - orientações sobre usabilidade. Rio de Janeiro, 2002.

BEST, M. L.; SMYTH, T. N. Global/ Local Usability: locally contextualized usability in the Global South. In: DOUGLAS, Ian; LIU, Zhengjie. Global Usability. Springer, 2011.

BEVAN, N. What is the difference between the purpose of usability and user experience evaluation methods? Computer science, Sweden, 2009. Disponível em: https://pdfs.semanticscholar.org/cba7/4036995821ca560d31bf397c695a460a63a5.pdf. Acesso em: 01 maio 2019.

BEVAN, N. European Usability Support Centres: Support for a More Usable Information Society: In: EUROPEAN TELEMATICS: ADVANCING THE INFORMATION SOCIETY OF TAP ANNUAL CONCERTATION MEETING, 1998. Proceedings [...] Barcelona, february, 1998.

CHEN, Y.-H.; GERMAIN, C. A.; RORISSA, A. Defining Usability: How Library Practice Differs from Published Research. Portal: Libraries and the Academy, v. 11, n. 2, p. 599-628, 2011.

CYBIS, W.; BETIOL, A. H.; FAUST, R. Ergonomia e usabilidade: conhecimentos, métodos e aplicações. São Paulo: Novatec, 2010.

DEE, C.; ALLEN, M. A survey of the usability of Digital Reference Services on Academic Health Science Library web sites. The Journal of Academic Librarianship, v. 32, n. 1, january, p. 6978, 2006.

DELAPLAI, I. J. Collaction and feed-back of user experience. Journal of the Royal Aeronautical Society, v. 70, n. 663. p. 415, 1966.

DIAS, F. S. Avaliação de sistemas de informação: revisão de publicações científicas no período de 1985-2005. 2006. 160f. Dissertação (Mestrado em Ciência da Informação), Universidade Federal de Minas Gerais, Belo Horizonte, 2006.

DUMAS, J. S.; REDISH, J. C. A practical guide to usability testing (revised edition). Exeter, UK: Intellect, 1999.

DYER, H. The usability of the concept of prejudice. Psychometrika, v. 10, n. 3, p.219-224, 1945.

GALITZ, W. O. The Essential Guide to User Interface Design: an Introduction to GUI Design Principles and Techniques. 2. ed. New York: Wiley Computer Publishing, 2002.

GEGNER, L., RUNONEN, M.; KEINONEN, T. Oscillating between extremes: a framework for mapping differing views on user experience. In: INTERNATIONAL CONFERENCE ON DESIGNING PLEASURABLE PRODUCTS AND INTERFACES, 11., 2011. Proceedings [...] Italy, 2011. DOI: $10.1145 / 2347504.2347566$. 
HAAKSMA, T. R.; JONG, M. D. T.; KARREMAN, J. Users' personal conceptions of usability and user experience of electronic and software products. IEEE Transactions on Professional Communication, v. 61, n. 2, p. 116-132, jun. 2018.

HASSENZAHL, M.; TRACTINSKY, N. User experience - a research agenda. Behav. Inf. Technol., v. 25, n. 2, p. 91-97, 2006.

ISO 13407: 1999. Human-centred design processes for interactive systems. 1999.

ISO 9126. Software product evalution: quality characteristics and guidelines for their use. 1991.

ISO 9241-210: 2010. Ergonomics of human system interaction - Part 210: Human-centred design for interactive systems Ergonomie de l'interaction homme-système, Partie 210: Conception centrée sur l'opérateur humain pour lês systèmes interactifs, Switzerland, 2010.

KUROSU, M. Usability, quality in use and the model of quality characteristics. In: KUROSU, M. Human-Computer Interaction: design and evaluation. New York, USA: Springer, 2015, p. 227-237.

LAW, E.; SCHAIK, E. V. Modelling user experience - an agenda for research and practice. Interacting with Computers, v. 22, n. 5, p. 313-322, sept. 2010.

LAW, E. L.-C. et al. Understanding, Scoping and Defining User eXperience: a survey approach. In: PROCEEDINGS OF ACM CHI 2009 CONFERENCE ON HUMAN FACTORS IN COMPUTING SYSTEMS, 2009, Boston. Anais [...]. Boston, 2009. p. 719-728. DOI 10.1145/1518701.1518813.

LOCKWOOD, B. Acting upon user experience - civil and military systems compared. Journal of the Royal Aeronautical Society, v. 70, n. 663, p. 418, 1966.

LUKESH, J. S. The effect of imperfections on the usability of quartz for oscillator-plates. American Mineralogista, v. 30, n. 5-6, p. 291-296, 1945.

MCNAMARA, N.; KIRAKOWSKI, J. Functionality, usability, and user experience: three areas of concern interactions. Interactions, v. 13, n. 6, p. 26-28, 2006.

MORVILLE, P. User Experience Desing. 2004.Disponível e: http://semanticstudios.com/user experience design/. Acesso em 03 fev. 2019.

NIELSEN, J. Designing Web Usability: The Practice of Simplicity. Boston, USA: Academic, 2008.

NIELSEN, J. Usability engineering. Boston: Academic Press, 1993. 
NORMAN, D.; MILLER, J.; HENDERSON, A. What you see, some of what's in the future, and how we go about doing it: $\mathrm{HI}$ at Apple Computer. 1995. In: CONFERENCE COMPANION ON HUMAN FACTORS IN COMPUTING SYSTEMS, New York, NY, USA, 1995.

PACK, T. Fiddling with the Internet Dials: Understanding Usability, 2003. Disponível em: http://www.infotoday.com/online/mar03/pack.shtml. Acesso em: 01 maio 2018.

SANTA ROSA, J.G.; PEREIRA JUNIOR, A.; LAMEIRA, A. P. Neurodesign: o cérebro e a máquina. Rio de janeiro: Rio Book's, 2016.

SCOPUS, 2019. Disponível em: https://www.scopus.com/home.uri. Acesso em: 14 jan. 2019.

SHACKEL, B. Usability: Context, framework, definition, design and evaluation, Interact. Comput., v. 21, n. 5,6, p. 339-346, 2009.

SILVA FILHO, A. M. Avaliação de Usabilidade: Separando o joio do trigo. Revista espaço acadêmico, ano 10, n. 112, set., 2010.

STARKER, L .N. User Experiences with Primary Journals on 16-Mm Microfilm. Journal of Chemical Documentation, v. 10, n. 1, p.5-6, 1970.

TULLIS, T.; ALBERT B. Measuring the user experience. Amsterdam: Morgan Kauffman, 2008.

USABILITY PROFESSIONALS ASSOCIATION, 2002. Disponível em: https://www.interactiondesign.org/literature/publisher/usability-professionals-association. Acesso em: 02 maio 2019.

VASCONCELOS, P. F. C. Use-user experience: uma metodologia de conhecimento das experiências dos usuários com base na etnografia. 2007. 146f. Dissertação (Mestrado em Informática Aplicada), Universidade de Fortaleza, Fortaleza, 2007.

W3C. What is driving accessibility?, 2002. Disponível em: https://www.w3.org/2002/Talks/0104-usabilityprocess/slide5-0.html. Acesso em: 21 jun. 2019.

WEB OF SCIENCE, 2019. Disponível em: https://login.webofknowledge.com/error/Error?Error=|PError\&PathInfo=\%2F\&RouterURL=h ttps\%3A\%2F\%2Fwww.webofknowledge.com\%2F\&Domain=.webofknowledge.com\&Src=IP\& Alias=WOK5. Acesso em: 02 maio 2019.

YOON, SO-YEON; LAFFEY, JAMES; OH, HYUNJOO. Understanding usability and user experience of web-based 3D graphics technology. International Journal of human-computer interaction, v. 24, n. 3, p. 288-306, 2008. 